\title{
Panorama futuro de la investigación en el campo del cemento
}

Prof. Dr. JOSE CALLEJA, Vicedirector del IETCC

\section{GENERALIDADES: La investigación pura y la aplicada}

Al tratar de meditar a solas primero y de transmitir a los demás después, las ideas surgidas acerca de lo que en criterio del opinante puede o debe ser la investigación en el campo del cemento, con vistas al futuro, no habría más remedio que erigirse en expositor y en crítico.

$\mathrm{Y}$ así sería preciso, siempre a título personal, hacer un recorrido de lo que ha sido y es (acaso sin deber serlo), y de lo que debió y debe ser (quizás sin serlo ni haberlo sido), la investigación en dicho campo.

Pero aún antes de esto, sería menester tratar de puntualizar sobre la investigación en sí, digamos que en abstracto.

Y la primera puntualización es la relativa a las clasificaciones tan arbitrarias, convencionales y artificiosas como paradójicamente aceptadas de la investigación, la más manejada de las cuales es la que hace dicotomía de la misma en pura, básica, teórica, fundamental, académica o "universitaria", y en aplicada, práctica, técnica, industrial o de desarrollo. Para simplificar podría decirse que, a juicio de los dicotomistas, la primera busca, ante todo, el conocimiento; y que la segunda persigue primordialmente la utilidad.

$\mathrm{Y}$ aquí viene la primera cadena de afirmaciones lapidarias: no hay conocimiento ni saber del que no pueda obtenerse pronto o tarde algún beneficio, pues los únicos conocimientos que jamás se aplican son los que no se tienen; ni se deja de ser práctico por poseer una amplia base teórica, sino, a lo más, por no usarla, o por usarla mal.

Esto quiere decir que no hay, que no debe haber conflicto entre Ciencia y Técnica, o entre Ciencia o Investigación pura y Ciencia o Investigación aplicada. Esta es consecuencia de aquélla y aquélla es condición sine qua non para que se desarrolle ésta. La ciencia o investigación pura de una época, más o menos tarde sirve de base firme e insustituible a la ciencia e investigación llamada aplicada de épocas posteriores, en una especie de carrera de relevos en la que circunstancialmente una u otra ocupan el puesto de vanguardia, cuando no marchan en paralelo. En todo caso, el conocimiento es anterior a la aplicación, e indispensable para ella.

Hecha esta puntualización se comprenderá el sentido y la intención con que en lo que sigue se trata de ambas investigaciones en el campo del cemento. 


\section{INTRODUCCION: La investigación en el campo del cemento}

Que la investigación aplicada o tecnológica requiere siempre un soporte de conocimientos básicos (que hay que adquirir si no se tienen) es innegable.

Por ejemplo: Desde un punto de vista técnico, esto es, práctico, se considera que la lentitud del fraguado y del endurecimiento del cemento portland es un inconveniente que hoy dificulta el desarrollo de la prefabricación y de la industrialización de la construcción y que, en su día, podría implicar el riesgo de que el hormigón perdiera la primacía en alguna de sus aplicaciones actuales, frente a posibles materiales y técnicas nuevas del futuro.

Solución: Investigar la forma de acelerar el fraguado y el endurecimiento del hormigón.

Modos: 1) Añadir al cemento "algo" que lo consiga; 2) calentar el hormigón (ya que calentado "todo va más deprisa").

Pero estas variantes de solución son empíricas. Se podría estar años enteros añadiendo "cosas" al cemento sin que se acelerase su hidratación. En todo caso, de encontrar algo que lo consiguiera, sería por casualidad, y no se sabría porqué, ni cómo, ni cuándo (es decir, ni si siempre y en cualesquiera condiciones se obtendría el mismo efecto). Y aún así, ese "algo" podría ser inoperante, por escaso, o por caro, o por incompatible en otros aspectos. Del mismo modo se podría calentar el hormigón de mil maneras sin conseguir sino una fracción, y no grande, de la resistencia que normalmente es capaz de tener.

Pero si se conoce la naturaleza del cemento anhidro - sus componentes- y su evolución en contacto con el agua -el mecanismo de su hidratación-, se puede: en el primer caso, ensayar productos abundantes, fáciles, baratos, compatibles, cuya posible acción esté prevista en el sentido deseado (así surgió la idea de emplear cloruro cálcico como acelerador); y en el segundo caso, se pueden ensayar desde el principio tratamientos térmicos que, sin desecar el hormigón ni forzar demasiado su hidratación, permitan lograr rápidamente porcentajes elevados de su resistencia máxima alcanzable (así se llegó a establecer el período de curado previo normal mínimo y los límites para los ritmos de calefacción y enfriamiento, o las presiones y tiempos óptimos de los tratamientos en autoclave).

La vía empírica - la que tantea sin orientación ni rumbo- es mucho más larga, costosa, insegura e ineficaz; pero es la única a la que pueden acceder los "aficionados" a la investigación. La vía racional -la que sigue el método científico- es la más corta, directa, eficaz y segura; naturalmente que ésta exige vocación en vez de, o además de, afición; sacrificio y formación permanente, actualizada y de vanguardia, en los principios y en la práctica de la pesquisa básica. No es para todos.

Por otra parte, una investigación acabada no agota un tema (ni siquiera un aspecto parcial o específico del mismo). Comoquiera que la investigación se autoalimenta y se realimenta de sus propios hallazgos, resulta - y es resultado bastante general- que los frutos de la investigación sobre un tema, lejos de eliminar éste, lo amplían. Es lógico: una montaña quita la visión del valle que está detrás. Pero su escalada no sólo no limita la panorámica anterior, sino que la amplía hasta lontananza, dejando ver un largo camino para alcanzar el horizonte visible que, una vez alcanzado, deja al descubierto otro nuevo, y así de forma interminable. La investigación reclama continuidad, profundidad y perseverancia, todo lo contrario de dispersión, superficialidad y diletantismo. También por eso no es apta para cualquiera. 
Quienquiera que diga, por ejemplo, que ya se ha estudiado bastante o incluso demasiado el fraguado del cemento, no puede hacerlo si no es por soberbia, por ignorancia o con intención; pero en todo caso, deliberada o ingenuamente, se equivoca como se verá por los hechos.

Se ha formulado la pregunta, a través de comités ad hoc para la investigación en el campo del cemento, de qué clase de investigación se necesita. Casi siempre se ha respondido, no con la que se necesita sino con la que se desea. $\mathrm{Y}$ se desea, en general, por parte de los seudo-investigadores, la más rentable, la que dé (o ahorre) más dinero en el más corto plazo posible. En realidad no se quiere investigación, se quiere algo tan contrario a la investigación misma, como es la taumaturgia.

Pero ¿qué se entiende y qué debe entenderse por investigación rentable? Lo que se entiende, lo que se quiere entender, está muy claro: algo que exija poco esfuerzo y tiempo y cueste poco dinero, que sea fácil de realizar y que dé los mejores resultados (económicos antes que técnicos) de un día para otro; lo dicho: taumaturgia, milagro. Pero lo que se debe entender es otra cosa, partiendo de la base de referir la rentabilidad de la investigación a un plazo corto, medio o largo, y a condición de definirla de una cierta manera; por ejemplo, por la relación costos/beneficios. Establecer los costos de una investigación es casi siempre posible y generalmente fácil, pues se pueden expresar en dinero (personal, equipos, etc.); pero determinar, y más aún, predeterminar sus beneficios, casi nunca es fácil, y muchas veces es muy difícil o incluso imposible. Además, no siempre estos beneficios son reducibles a pesetas. $\mathrm{Y}_{¿}$ cómo, entonces, establecer una relación entre dos magnitudes heterogéneas, una de las cuales por añadidura queda sin precisar? A esto hay que agregar que, hablando en general, aunque de alguna manera se pueda fijar una repercusión económica de la investigación, ésta no es, ni con mucho, la más importante en todos los casos. Por ejemplo: el descubrimiento de las penicilinas o de la vacuna Salk, por no citar sino fármacos modernos, ¿qué frutos ha dado? ¿Se computan éstos por el dinero que con dichos descubrimientos gana la industria farmacéutica mundial, lo cual es fácil, o se utiliza como parámetro el incalculable bien que tales fármacos han aportado, aportan y aportarán a la humanidad? Porque si este bien expresable en dinero -es un decir- es cero, el cociente costo/beneficios de la investigación para realizar tales descubrimientos, y con él la rentabilidad de la misma, vienen a ser infinitos, resultando que en este caso, como en otros muchos, dista bastante de ser absurdo. Absurdo sería, en cambio, considerar dicha rentabilidad nula, si se definiese por la relación inversa beneficios/costos, asignando a aquéllos —en pesetas, claro está- el valor cero.

No hay más que pensar en las horas de trabajo que en el mundo y al año se dejan de perder, al acortarse el número y la duración de las bajas por enfermedad, mediante el empleo de antibióticos. Pero ¿quién piensa en ello, si no redunda en algo tangible para una determinada individualidad?

Lo que sucede y no deja de ser paradójico, es que, en general, lo evaluable en dinero, lo tenido por rentable, lo es para uno o para pocos bien determinados; mientras que lo no traducible —directa e indirectamente - a pesetas, lo tenido a veces por no rentable, suele beneficiar a la totalidad o a una gran mayoría imprecisa. Así pues, se contabiliza lo poco concentrado y se deja de contabilizar lo mucho repartido.

\section{PASAdo, PRESENTE Y FUTURo DE LA INVESTIGACION EN EL CAMPO DEL CEMENTO}

El Grupo de Enlace y Coordinación de la Investigación, del CEMBUREAU, patrocina los esfuerzos cooperativos, tanto en la ciencia y en la investigación exploratorias a largo pla- 
zo, como en la investigación aplicada que comprende la mayor parte de la dedicada a la industria del cemento.

Ahora bien, para que la investigación redunde en favor de la promoción de un producto o material, es indispensable tener, y en su caso adquirir, nuevos conocimientos que pueden ser aplicados a las mejoras de tipo práctico y a la innovación.

Empecemos analizando la situación pretérita de la investigación básica sobre cemento y hormigón.

En el pasado, y a escala internacional, los institutos de investigación sobre hormigón y cemento funcionaban como centros destacados en la pesquisa básica y exploratoria, manteniendo contactos con la industria y asegurando una comunicación eficaz, con frecuencia incluso entre la investigación fundamental y los problemas de la práctica real y cotidiana. De tal manera, que dicha investigación era reconocida como base amplia para el progreso de la industria del cemento y del empleo de éste en la construcción y en las obras públicas, gracias a la difusión y al libre intercambio de sus resultados.

En época más reciente se puede decir que la investigación académica en los campos del cemento y del hormigón se incrementó y expandió en la década de los 50, y que llegó a alcanzar una estabilización cuantitativa, en la mayor parte de los países, en el decenio del 60 al 70. Y también que, a partir de entonces, ha habido una regresión por lo que se refiere al interés de los sectores públicos en la investigación básica del cemento, primero en los Estados Unidos y después, quizás por mimetismo, en la Europa Occidental; pero no así en la Europa Oriental, ni en el Japón, y ninguna prueba mejor de ello que el VI Congreso Internacional de Química del Cemento de Moscú, en 1974, y el V de Tokyo, en 1968.

Ya en los tiempos actuales se ha llamado la atención, por parte de un grupo de trabajo, dentro del Grupo de Coordinación y Enlace del CEMBUREAU, sobre el hecho de que la investigación básica de interés para la industria del cemento está corriendo hoy un serio y grave riesgo de fragmentación y aislamiento, mayor que el de dicha investigación en otros campos industriales. $\mathrm{Y}$ asimismo se ha señalado la tendencia de los organismos públicos de investigación en los últimos años a conservar la tecnología y metodología existentes, frenando otras nuevas, cuyo desarrollo podría contribuir a una extensión de las aplicaciones y del mercado del cemento.

Esto se ha manifestado notablemente en los últimos congresos sobre cemento y hormigón, y concretamente en el de Moscú antes citado, en los que se ha apreciado un descenso del progreso científico, con aumento de hallazgos duplicados, e incluso multiplicados, en lo que se refiere a investigación tecnológica, poco relacionada, por otra parte, con el desarrollo de las aplicaciones del material cemento.

En resumen: Existe el peligro de que la investigación científica de base en los campos del cemento y del hormigón languidezca y se desentienda de la investigación industrial aplicada y de desarrollo, la cual a su vez se vería privada del continuo y necesario debate con la primera. La consecuencia puede ser que cada una de ellas discurra cada vez más por caminos y con orientaciones distintas, no conectadas entre sí, y alejadas además de la innovación tecnológica con vistas a las exigencias reales del mercado del cemento en cada coyuntura.

¿Qué hacer para evitar este peligro y sus graves consecuencias a medio y largo plazo? El Grupo de Enlace y de Coordinación de la Investigación del CEMBUREAU propone por sí y para sí las siguientes soluciones:

1. La organización, siempre a escala internacional, de reuniones sobre posibilidades de una investigación fundamental avanzada que pudiera servir de base para, o convertirse en, una investigación aplicada de desarrollo e innovación al cabo de unos años; 
investigación que sería realizada por los sectores industriales y/o por los institutos de investigación del ramo, pero indispensablemente por estos últimos.

2. La comunicación con otros organismos de estudio - por ejemplo, la RILEM, etc.para tratar de intensificar esfuerzos de todo tipo en apoyo de su trabajo encaminado al mismo fin, así como para llegar a un entendimiento acerca de:

i) lo que no se sabe, pero que puede llegar a ser explotado a base de estudios que no requieran investigación;

ii) lo que no se sabe y necesita de la investigación básica para su conocimiento y desarrollo.

3. Planteamiento y discusión de problemas críticos o valiosos para la promoción del cemento, tanto desde el punto de vista del desarrollo tecnológico posible a largo plazo, como para la extensión o la defensa de su mercado a plazo corto.

\section{LA NECESIDAD DE UNA CIENCIA DE LOS MATERIALES, ESPECIFICA PARA EL CEMENTO}

Todo el mundo implicado en el tema está de acuerdo en la necesidad de una llamada "Ciencia de los Materiales". Pero bien entendido que referida ésta a la escala molecular y atómica, pues éste es muchas veces el único camino de estudiar, suficientemente a fondo, la composición, la naturaleza y la estructura de los materiales, cuyo conocimiento lleva a comprender las causas del comportamiento de éstos y de determinadas diferencias en dicho comportamiento. En una palabra, la Ciencia de los Materiales se concibe, y no hay otra manera de hacerlo, como una ciencia básica o fundamental, de naturaleza física y química.

Por ella se ha llegado ya a comprender, por ejemplo, por qué cuando se utiliza menos agua en el hormigón éste tiene mayor resistencia, y por qué esto se logra sin detrimento -o incluso con mejora de la trabajabilidad-, mediante la acción de ciertos productos orgánicos. Por el mismo camino nada impide, en principio, que, conocida a fondo a través de la Ciencia de los Materiales la influencia de las formulaciones en los cambios de comportamiento del cemento, se puedan obtener, por ejemplo, aditivos para cada necesidad tecnológica que se nos plantee.

De donde se deduce una vez más que no se puede prescindir en absoluto de la ciencia básica en la investigación tecnológica, pues la primera fue, es y será siempre anterior a la segunda. La ciencia básica crea conocimiento; la tecnología lo aplica. No se puede aplicar lo que previamente no se posee o adquiere.

Estas ideas son sustentadas, entre otros muchos no sospechosos de teorizantes, por Bryant MATHER (*), interesado en cuestiones de normas en general y de cemento en particular, como Presidente que es de la ASTM, pero responsable también ante el Cuerpo de

(*) Presidente de la ASTM.

Director del Laboraforio de Hormigón de la U.S. Army Engineers. Waterways Experiment Station, Vicksburg, Miss. U.S.A. 
Ingenieros de su Organización, de la gestión de un programa de investigaciones en el campo del hormigón. Hasta tal punto, que lamenta la supresión de la antigua División de Ciencia de los Materiales, de la ASTM; pues si bien reconoce que la ciencia básica o fundamental sobre cemento no se integra en la elaboración de normas, entendiendo por tales lo que se exige para comprar y vender cemento, desde el punto de vista de su calidad, no deja de reconocer que las normas fatalmente tienen que apoyarse en métodos de ensayo, y que éstos deben estar en constante depuración y simplificación. Y que, para ello, es indispensable llevar a cabo la oportuna investigación básica que ponga de relieve las relaciones entre los principios fundamentales y los ensayos simplificados; que permita tener ideas y reunir suficientes datos para establecer estos métodos, así como, en definitiva, para mejorar los medios de prever el comportamiento final de los materiales -en su caso del cemento-, con objeto de poder formular exigencias razonables y defendibles, y de estipular límites en las normas.

Y se pregunta MATHER: ¿quién toma en U.S.A. —de no ser como antes la propia ASTM - la iniciativa de este tipo de investigación indispensable para el progreso de la normalización? La respuesta parece ser: los organismos públicos, los centros de investigación y la propia industria. Otro punto decisivo es el de la coordinación y reparto de misiones, en función de la especificidad y características peculiares de cada uno de estos estamentos.

Se entiende también que ésta que se ha dado en llamar "Ciencia de los Materiales" ha de servir de base y de plataforma de lanzamiento para el desarrollo y la innovación de la correspondiente tecnología.

Una de las mejores formas de hacerlo, por no decir la única, es, como se indicaba, permitir la predicción del comportamiento de cualquier material, en cualesquiera condiciones de servicio. Esto, que en general, apenas se ha conseguido en algún caso, y no de forma completa o satisfactoria, menos aún se ha logrado en el caso de los cementos. $\mathrm{Y}$ ello es porque existe un fallo o vano entre el grado de conocimiento de la naturaleza de los cementos, y el de los procesos de su formación, transformación y deterioro, lo cual impide prever los efectos y las consecuencias de cualquier cambio en el sistema. Por eso cada nuevo material cementicio, o cada modificación de uno viejo, cada nueva aplicación de uno u otro a casos y en condiciones específicas distintas, exigen ensayos previos para determinar la correspondiente idoneidad.

Por eso también, es innegable que si una hipotética ciencia de los materiales aplicada al cemento hubiera de llenar el vacío antes mencionado, para así contribuir al desarrollo de la técnología, no podría de ningún modo hacerlo sin recurrir, mediante investigaciones y estudios básicos, a un mejor (por más profundo y armonizado) conocimiento, tanto de la naturaleza del cemento - anhidro e hidratado- en sí, como de los procesos de transformación o reacción en su fabricación y aplicaciones.

No solamente no se ha llegado a la necesaria profundidad, armonía y paralelismo en tales conocimientos, sino que aún se está lejos de la meta. Es sabido que cementos, no ya de diferentes orígenes, sino de uno determinado, es decir, un solo y mismo material, se comporta de modo distinto en cuanto a velocidad de hidratación, fraguado, viscosidad, resistencia, respuesta frente a aditivos, etc., en función, tal vez, de su historial térmico, de la forma y tamaño de sus partículas, de su superficie específica, de su composición química (naturaleza y cantidad de componentes mayoritarios y minoritarios), de la cristalinidad y defectos de red de sus especies, del tiempo y condiciones de su ensilado, etc. Es decir, de condiciones y factores físicos y químicos, intrínsecos y extrínsecos. Pues bien, no hay, por ahora, medio de conocer y fijar todos estos factores, ni siquiera de enumerarlos, para determinar de modo razonable el comportamiento de un cemento, y esto hace que sea difícil —e improcedente muchas más veces de las que se piensa- la compa- 
ración de resultados de un mismo material, obtenidos por distintos operadores o laboratorios. Y, en consecuencia, también son improcedentes, injustificadas y no válidas las conclusiones que de tal tipo de comparaciones se pretenda extraer.

Aparte de ello, las definiciones y los conceptos básicos generalmente bien establecidos en apariencia, y convencionalmente aceptados, o no están suficientemente esclarecidos, o se hallan en trance de revisión. Son muchos los aspectos que, ficticiamente superados, requieren aún mucho estudio e investigación. Tal sucede con la idea o noción de reacíividad. Por ejemplo, la transformación $\mathrm{C}_{3} \mathrm{~A} \rightarrow \mathrm{C}_{3} \mathrm{AH}_{6}$ se retarda o se inhibe con cantidades pequeñas o grandes, respectivamente de yeso o ligninsulfonatos. A la inhibición se le sigue llamando retardo y considerando como tal, con olvido de la formación de sulfoaluminatos hidratados $\mathrm{y}$ de ligninsulfonatos complejos, en lugar de $\mathrm{C}_{3} \mathrm{AH}_{6}$.

Otro ejemplo lo constituye la distinta velocidad de hidratación del $\mathrm{C}_{3} \mathrm{~S}$ y del $\mathrm{C}_{2} \mathrm{~S}$, que parece poder tener una posible explicación indirecta en el "efecto Hedvall", según el cual la reactividad de un sólido aumenta durante alguna de sus transformaciones cristalinas, y como consecuencia de ella. Pero la reacitvidad de un sólido $\left(\mathrm{C}_{3} \mathrm{~S}, \mathrm{C}_{2} \mathrm{~S}\right)$ depende también de la materia prima de que proceda, así como de circunstancias y factores externos, sin que hoy se sepa a ciencia cierta por qué.

En suma, la hidratación y el endurecimiento del cemento han sido objeto de interés y controversia, desde el antiguo dilema: cristalización o formación de coloides (Le Chatelier-Michaelis), hasta el más moderno punto de vista: disolución y cristalización, o transformación topoquímica. Pero, en todo caso, se está casi tan lejos de explicar el fenómeno, como cuando hace cerca de sesenta años el propio Le Chatelier, defendiendo su teoría y atacando la contraria - y exagerando, sin duda, al hacerlo- hacía observar que, así como se suele decir que los cuerpos reaccionan por que tienen "mutua afinidad" (lo cual no es decir nada), algo semejante sucede con el endurecimiento basado en la formación de coloides, cuya definición se evade, pero que se aceptan como cuerpos capaces de endurecer. Con lo cual se llega más o menos, a la perogrullesca conclusión de que los cementos endurecen porque tienen la propiedad de endurecer. Que en este terreno, y precisamente a partir de lo más básico se puede hacer mucho y es imprescindible hacerlo, si se quiere avanzar en tecnología, es indiscutible.

Otro campo en el que los avances básicos pueden sacar del empirismo en que se está inmerso, es el de los aditivos, hasta poder predecir cualitativa y cuantitativamente sus efectos en todas circunstancias. Por ejemplo, las diversas teorías emitidas acerca de la acción de los aceleradores y retardadores explican sólo parte de los hechos, probablemente porque la acción de un aditivo sencillo es múltiple -y mucho más la de los aditivos compuestos-.

Todas las comparaciones y conclusiones basadas en la relación de una variable estudiada con la superficie específica de componentes anhidros o hidratados, sobre todo en su aspecto cuantitativo, son dignas de ponerse en cuarentena, pues dicha superficie depende del método y condiciones de la medida, y de la evolución y vicisitudes - historia de la preparación, tratamiento y conservación de las especies o materiales sometidos a estudio-. Del mismo modo, diferencias en el tamaño de las partículas pueden inducir, al aplicar algunas técnicas, a interpretar como alotropía o polimorfismo lo que no lo es o, al menos, a hacerlo cuando es dudoso. Y, en relación con ambos, a atribuir energías, propiedades y comportamientos ficticios a determinadas especies químicas y a los materiales que las contienen.

La importancia tecnológica de los cambios de volumen - y su medida - ligados a la hidratación es evidente. Pero estos cambios de volumen aparente y los parámetros de los que parecen depender, no guardan relación con el cambio de volumen real de las fases sólidas que intervienen, sino más bien con las condiciones de la hidratación y con la naturaleza de sus productos. Esto va de acuerdo con el punto de vista de que los cambios de volumen dependen de la facilidad de acomodación de los productos hidratados al formarse, 
densificando la estructura y sin ejercer grandes tensiones en ella, lo cual sucede en el cemento y explica su escasa expansión, en relación con la del yeso hemihidrato o la de la cal. Pero, en todo caso, hoy por hoy no es previsible en general, cuándo un cemento va a expandir o no al hidratarse.

En el caso de los productos hidratados del cemento, cuyo comportamiento sólo se podrá predecir asimismo cuando se conozcan con suficiente detalle y precisión, tanto sus propiedades fundamentales como los mecanismos de sus procesos de formación y transformación, la situación es aún más confusa y complicada.

En cuanto a composición química, la de los silicatos hidratados de la pasta del cemento portland no está aún bien conocida, y en particular el número de moléculas de agua ligadas a los geles tobermoríticos, así como las asociadas en diversas formas, incluso en los capilares. Tampoco están bien determinadas las relaciones $\mathrm{H} / \mathrm{S}$ y $\mathrm{C} / \mathrm{S}$ en la(s) fase(s) $\mathrm{C}_{\mathrm{x}} \mathrm{S}_{\mathrm{y}} \mathrm{H}_{\mathrm{z}}$.

En el caso de los cementos aluminosos las incógnitas no son menores, quedando por explicar el porqué de la influencia tan decisiva de la relación agua/sólido en las propiedades y comportamiento de sus pastas hidratadas. $\mathrm{Y}$ todo esto demuestra que la estequiometría de los cementos portland y aluminosos hidratados es variable, lo que es imprescindible tener en cuenta al comparar - y evaluar - resultados obtenidos de distintas preparaciones.

Por otra parte, la densidad de la pasta, uno de los principales factores de los que depende la porosidad, y que se relaciona con la durabilidad y con la resistencia, si bien se define simplistamente de una forma en apariencia bien especificada, resulta que no es un valor único, sino que depende del método con que se mida y del modo y condiciones en que se haga. Es decir, la definición "actual" de densidad es incompleta e insuficiente, a efectos comparativos y de correlación y valnración de resultados.

Algo semejante sucede con el área superficial o superficie específica de la pasta de cemento. En la hidratación del cemento anhidro se produce un gran desarrollo de superficie, pero ésta depende, no sólo de la naturaleza del material de partida, sino también del método y condiciones de la hidratación, y del procedimiento para determinarla.

Todo lo anterior constituye una serie - limitada y reducida- de ejemplos que abonan la tesis expuesta al principio: queda mucho por conocer acerca de las propiedades de los materiales y/o acerca de los mecanismos de sus transformaciones e interacciones; existe además, en general, un vacío entre uno y otro conocimiento, necesarios ambos para poder predecir, conforme a la finalidad última de una "ciencia de los materiales", el comportamiento de éstos en cualquier circunstancia.

Más concretamente por lo que al cemento hidratado se refiere, su comportamiento físicomecánico, se puede considerar agrupado en dos aspectos: el de los cambios de volumen y el de las resistencias.

Los cambios de volumen de toda naturaleza — físicos y/o químicos, positivos o negativos, reversibles o irreversibles- por principio deben ser mínimos.

La previsión de los cambios de volumen de los productos hidratados, debidos a su porosidad y a las variaciones de humedad es muy difícil, porque el secado y la deshidratación de los mismos no son procesos estrictamente sucesivos, sino parcialmente simultáneos: la deshidratación comienza antes de haberse producido el secado total; y, por otra parte, porque tales cambios afectan, en general, no sólo al volumen sólido de un sistema, sino al volumen total del mismo.

Los cambios de volumen se determinan y se refieren a condiciones que se llaman "secas" o "húmedas", pero que no están suficientemente bien definidas en cuanto al contenido de humedad del sistema, ni —aún estándolo- son representativas de las condiciones prác- 
ticas y reales de servicio del material. Así, los resultados de estudios e investigaciones llevados a cabo sobre cambios de volumen debidos a la humedad, difícilmente son reproducibles, comparables y representativos, y por supuesto previsibles.

La expansión debida al hielo y sobre todo la parte irreversible de la misma, puede ser un índice del grado de deterioro del cemento y se relaciona con la porosidad de la pasta, con su grado de saturación, con las condiciones del enfriamiento, etc.; pero la predicción del deterioro depende de lo que se sepa acerca del comportamiento del agua adsorbida en los capilares más finos bajo la helada. Se cree que la congelación de un material poroso embebido a saturación crea una situación de exceso de agua, causante de la dilatación y del deterioro, al que contribuye la congelación del agua exudada, en las microfisuras.

La expansión causada por ataque de sulfatos -y el correspondiente deterioro- se atribuyen a formación de ettringita a partir de éstos y del aluminato cálcico hidratado. Pero el mecanismo completo y real del ataque presenta aún muchos puntos oscuros. Por ejemplo: a) la contribución de la conversión $\mathrm{Ca}(\mathrm{OH})_{2} \rightarrow \mathrm{CaSO}_{4} \cdot 2 \mathrm{H}_{2} \mathrm{O}$ al proceso expansivo; b) el carácter topoquímico o no del mismo; c) la paternidad del trisulfoaluminato o del monosulfoaluminato en cuanto a la expansión; d) en el primer caso, la naturaleza cristalina o geliforme de la ettringita expansiva; e) el papel de la fase ferrítica en el proceso expansivo; f) la acción específica del $\mathrm{MgSO}_{4}$, atacando además a los silicatos, cosa que no hacen los sulfatos alcalinos; g) la insuficiencia de los métodos de ensayo acelerados para predeterminar la susceptibilidad de los cementos a los sulfatos, y tantos otros.

La carbonatación produce retracción entre HR $25 \%$ y HR $100 \%$, máxima a HR $50 \%$, por un mecanismo no bien conocido. Por otra parte, ejerce, según qué circunstancias, efectos benéficos o detrimentales, pero en todo caso se debe evitar o controlar, por su influencia en los resultados y en la falta de correlación entre los hallados en distintos laboratorios, acerca de un mismo problema. Esto no siempre -en realidad pocas veces- se tiene en cuenta, y así se ha considerado durante mucho tiempo como $\alpha-\mathrm{C}_{4} \mathrm{AH}_{13}$ lo que no es sino un producto contaminado por carbonatación, que responde a $\mathrm{C}_{3} \mathrm{~A} \cdot \mathrm{CaCO}_{3} \cdot 11 \mathrm{H}_{2} \mathrm{O}$.

La resistencia, como criterio práctico para determinar el empleo del cemento, es imprevisible. Se sabe que, en general, un sistema es tanto más resistente, cuanto menos poroso es y cuanto mayor perfección cristalina posee. Cuál sería la resistencia teórica máxima de la pasta de cemento para una porosidad cero y una máxima cristalinidad, es algo imposible de predecir con cualquier grado de aproximación, ya que se trata de un sistema complejamente poroso, heterogéneo y parcial e imperfectamente cristalino, en el que las uniones probablemente se realizan por una combinación de fuerzas físicas y químicas (de van der Waals, por puentes de hidrógeno, de adsorción, de valencia, de polimerización de silicatos, etc.), cuya acción y participación es desconocida.

Por otra parte, las hipótesis que pudieran establecerse para intentar el cálculo de dicha resistencia teórica estarían tan alejadas de la realidad, y en un grado tan impreciso, que los resultados no tendrían ningún valor.

También se ha pensado que la resistencia de la pasta depende en gran manera del desarrollo de superficie interna y de la naturaleza de la misma en la hidratación, del grado de ésta, de una posible estructura laminar o fibrosa, o incluso de una configuración altamente coloidal de los productos hidratados, así como de diversas interconversiones de unas fases en otras.

En resumen, las propiedades mecánicas, y entre ellas la resistencia, son una complicada función de multitud de variables complejas e interrelacionadas, que, ni todas son conocidas, ni todas las que lo son, en realidad o en apariencia, pueden ser medibles y controlables. 
He aquí, pues, otro campo más que suficiente para el desarrollo de una ciencia del cemento, como uno de los capítulos importantes de una más amplia y general ciencia de los materiales. Y no seamos optimistas o ingénuos, en todo caso simplistas, preguntando cuándo podrán estar resueltos todos estos problemas, porque quizás la única respuesta prudente y sensata sería la de nunca.

Para el establecimiento de un tal capítulo de la Ciencia de los Materiales, las mayores dificultades estriban en que:

1. Las definiciones de muchos de los conceptos básicos que se manejan, aparentemente completas en general, no lo son en el caso particular del cemento, y aún en el de los componentes anhidros y/o hidratados del mismo. Así sucede con los conceptos de densidad, superficie específica, tamaño y forma de partículas, cambios de volumen, etc., cuyos valores dependen de una serie de factores, ni todos conocidos en cuanto a número, ni todos tenidos en cuenta o controlados, pero en cualquier caso no incluidos en las respectivas definiciones. De donde se deduce que más que de una definición, se precisa en tales circunstancias de una especificación, si se quiere evitar el riesgo -y sus consecuencias - de comprar resultados no comparables, de hacer valoracciones equivocadas e interpretaciones erróneas de las mismas y, finalmente, de extraer conclusiones falsas.

2. Ni el concepto de reactividad, ni los factores que en cada caso influyen en la velocidad de hidratación, ni en el curso que ésta sigue y la cinética y estequiometría por la que se rige, ni la composición química de los productos de partida y/o de reacción en general, están establecidos con suficiente precisión en la actualidad.

Una posible solución para el caso, en el seno de una verdadera ciencia de los materiales aplicada a los cementos, sería dirigir en el futuro - a partir de ya mismo - los estudios y las investigaciones a escala mundial, sobre bases y con orientaciones comunes, aceptadas con la máxima generalidad posible. Dicho de otro modo, habría que llegar al establecimiento lo que se podría llamar una "normalización" o sistematización de la investigación, con especificación lo más completa y detallada posible sobre definiciones de: conceptos, parámetros, magnitudes, procesos, métodos de ensayo, procedimientos y condiciones operatorias, y de cuantas variables y factores puedan influir en la reproducibilidad de resultados y en la valoración y comparación de los mismos. Y esto, de la misma manera que se normaliza y se establecen prescripciones y especificaciones sobre materiales y métodos de ensayo tecnológicos, terreno en el que ya se tiende a llegar — vista su necesidada una armonización y a una homologación de acatamiento general.

\section{NUEVAS PREVISIONES ACERCA DE LA INVESTIGACION EN EL CAMPO DEL CEMENTO Y DE LA EVOLUCION DE SU INDUSTRIA}

El título de este apartado me retrotrae, al del que, con el mismo número, desarrollé en el trabajo titulado "Lo que el futuro promete y exige al cemento y al hormigón", y que fue presentado en la II Asamblea General del IETCC, pronto hará dos años. Ambos títulos difieren en su amplitud y en el adjetivo "nuevas", y por lo tanto no voy a repetir aquí lo allí expuesto, aún cuando prácticamente en su totalidad sigue siendo válido.

Se podía decir entonces, y se puede reafirmar aún con más rotundidad ahora, que las orientaciones de la investigación ya presente y más aún futura, así como la evolución de la industria en el campo del cemento, están condicionadas por la crisis energética y por la económica. 
En el aspecto energético la crisis actual del petróleo, de los combustibles y de la energía en general, afecta a toda la industria y en particular a la pesada, en todos los países.

Con previsora intuición, el Consejo del Atlántico Norte creó hace siete años el Comite para el Estudio de las Exigencias de la Sociedad Moderna, con el fin, entre otros, de realizar una serie de proyectos dentro del Programa sobre la "Utilización Racional de la Energía". Uno de éstos, el llamado Proyecto Básico de Datos Industriales Internacionales, propuesto por los Estados Unidos, tenía por objeto reunir información sobre el consumo energético de las grandes industrias, comenzando por dos de ellas: la del acero y la del cemento.

El informe relativo a esta última, en el que han participado diversos paises europeos y organismos internacionales, ha quedado terminado en agosto pasado y será dado a conocer en el curso del presente otoño. Tal vez en estos momentos sea ya, o esté a punto de ser, del dominio público.

De todos modos, es sabido que el informe concluye con una serie de recomendaciones acerca de los proyectos de investigación y desarrollo que se deben abordar para conseguir, con gran probabilidad de éxito, una mejora de rendimiento energético por parte de la industria cementera.

Los proyectos recomendados comprenden los estudios siguientes:

i) de las propiedades y de los límites de utilización de los cementos que contienen componentes secundarios (en el nuevo Pliego español RC-75 serían los cementos PA y los C, aparte de otros posibles, no contemplados en él);

ii) de la influencia de la finura y de la granulometría del cemento en las propiedades del hormigón;

iii) de la influencia de los coadyuvantes de molienda del clínker sobre las propiedades del hormigón, y de la normalización de dichos productos;

iv) de la influencia de la finura y de la granulometría de las materias primas en la fabricación de cemento;

v) de las reacciones entre áridos y álcalis;

vi) del empleo de la precalcinación y de los precalcinadores para poder utilizar los intercambiadores de calor de cuatro etapas en la fabricación de cementos de bajo contenido de álcalis, incluso a partir de materias primas con contenidos de álcalis relativamente altos;

vii) de la fijación de álcalis en los polvos de los hornos;

viii) de la influencia del contenido en sulfatos del clínker sobre su aptitud a la molturación;

ix) de las restricciones impuestas a la adición del yeso por el contenido de sulfatos del clínker, así como de la posibilidad de sustituir eventualmente el yeso como adición al mismo.

El Comité antes citado y el Grupo de Estudio sobre cemento del CEMUBUREAU recomiendan abordar estos temas a niveles nacionales, trabajando concertadamente, y así se lo han hecho saber a la Agencia Internacional para la Energía. 
Como se ve, los proyectos señalados cubren bien los campos de la fabricación y de la utilización del cemento, en cuanto que interesan:

a) a las materias primas — proyectos i), iv), ix)—;

b) a los procesos:

a) de molienda — proyectos ii), iii), iv), viii)—;

ß) de cocción - proyectos vi), vii)-;

c) a las normas —proyectos i), ii), ix)-;

d) al hormigón —proyectos i), ii), iii), v)—.

Es tal vez interesante consignar aquí que el IETCC no es ni ha sido ajeno a estas inquietudes, ya que desde el año pasado se ha planteado un programa de investigación, actualmente en marcha, el cual incluye el proyecto i) y parte de algunos otros, entre ellos el ix) en combinación con el propio i). Asimismo está interesado de forma que se pudiera llamar tradicional, en algunos matices específicos de los problemas que plantean los temas vi) a ix).

Dentro de las consideraciones antes señaladas, una de ellas, la de modificar los hornos con precalentadores, ya es una realidad conseguida primero en el Japón y adoptada después en otros países, a base de reducir las cámaras de clínkerización - proceso IHI-SF o similares-, de la cual se ha hablado y discutido en estos Coloquios. Otra alternativa que alguien ha apuntado en alguna ocasión, es la de utilizar sistemas intercambiadores con más etapas, e incluso con menos etapas para materiales con contenidos altos de azufre y álcalis.

De cualquier modo es preciso no olvidar que la economía térmica en los hornos de vía seca no es gratuita, y que exige pagar el precio de una mayor concentración de álcalis en el clínker, con tendencia al falso fraguado; de la formación de incrustaciones y anillos; de la corrosión de los filtros; y de un mayor castigo del refractario. Tributo que, sin duda alguna, los nuevos sistemas perfeccionados, u otros que puedan surgir, anularán o reducirán sustancialmente.

En punto a facilidad de proceso y a calidad de producto (sobre todo a la primera), se depurará el conocimiento que ya se tiene acerca de las variables que influyen en el tamaño de los gránulos del clínker y en la curva de distribución granulométrica del mismo, a través de la capacidad de aglomeración y de nodulización del crudo, y que habrá que saber y poder controlar.

En efecto, en la formación y tamaño de los gránulos de clínker intervienen favorablemente la aglomeración y la nodulización por formación de fase líquida, y desfavorablemente la formación y el crecimiento de los cristales y partículas de $\mathrm{C}_{3} \mathrm{~S}$.

La fase líquida actúa por sus fuerzas capilares - tensión superficial, capacidad humectante-, junto con el tamaño de las partículas y la velocidad del horno, en la formación de las gránulos.

Y en la cantidad de fase líquida interviene la composición química, a través del grado de saturación de cal y del módulo silícico del crudo (concretamente, de la alúmina, del hierro, de la magnesia y de los álcalis).

La fase líquida se forma en cantidad alrededor de los $1.350^{\circ} \mathrm{C}$ y aumenta poco a mayores temperaturas hasta $1.450^{\circ} \mathrm{C}$. 
El $\mathrm{C}_{3} \mathrm{~S}$ se forma también en este intervalo, a velocidad que aumenta mucho con la temperatura, lo cual favorece el rápido crecimiento cristalino y la fusión intercristalina, incrementando el tamaño de las partículas y entorpeciendo la nodulización. Esta cesa para un contenido crítico de $\mathrm{C}_{3} \mathrm{~S}$.

Por lo tanto, la nodulización se favorece en la zona del horno entre los $1.000^{\circ} \mathrm{y}$ los $1.450^{\circ} \mathrm{C}$. Esta debe ser lo suficientemente larga (más en la parte de calentamiento y formación de fase liquida $-1.100^{\circ}$ a $1.300^{\circ} \mathrm{C}$ - que en la de predominio de la formación de $\mathrm{C}_{3} \mathrm{~S}-1.300^{\circ}$ a $1.450^{\circ} \mathrm{C}-$ ) para que haya espacio y tiempo suficiente para la nodulización.

Hasta el punto de que el clínker pulverulento, escaso en fase líquida y con aspecto de piedra pómez, no nodulizado, es en su mayor parte un aglomerado de grandes partículas de cristales de $\mathrm{C}_{3} \mathrm{~S}$. Otra aglomeración de gránulos puede tener lugar en el enfriamiento, por solidificación de la fase líquida, pero ésta da lugar a clínker con aspecto de coque.

De todo ello se deduce que para conseguir una buena nodulización efectuada en suficiente espacio y tiempo, a base de una mayor cantidad de fase líquida a mayor temperatura, y $\sin$ alterar la formación de $\mathrm{C}_{3} \mathrm{~S}$, conviene trabajar con un módulo $\mathrm{A} / \mathrm{F}$ bajo. También se consigue reduciendo el módulo silícico o el grado de saturación — con aumento de la fase líquida en el primer caso y aumento o invariabilidad de la misma en el segundo-, pero con disminución del contenido de $\mathrm{C}_{3} \mathrm{~S}$ en ambos. $\mathrm{Y}$ también se logra rebajando la temperatura de la zona de máxima, cuando es muy alta, o reduciendo en $30-40 \mathrm{cal} / \mathrm{kg}$ el consumo específico de calor en la clinkerización, cuando la aptitud del crudo lo tolera.

Modificaciones adecuadas en el perfil de temperatura de los hornos tubulares, o en el diseño de éstos, así como el estudio de la acción de determinados fundentes y mineralizadores, pueden ayudar a resolver, asimismo, el problema para unos crudos dados. Todo ello puede merecer la pena, por cuanto que cambios de esta naturaleza, que facilitan la marcha del horno, hacen más viable la automatización y pueden permitir a veces aumentos de producción hasta de un 15 por ciento, mejorando incluso la calidad. Es de advertir que también esta línea de acción, al menos en alguno de sus aspectos, está presente en la actividad actual el IETCC.

En cuanto a los álcalis y a los sulfatos, tanto en el clínker como en el cemento, constituyen un tema digno de mención, típico de un capítulo de la Ciencia y de la Tecnología del cemento, por sus múltiples vertientes, tanto en la fabricación como en el empleo del material. En efecto, los álcalis y el $\mathrm{SO}_{3}$ de las materias primas y del crudo influyen en el proceso de clinkerización y por lo tanto en los sistemas de llevarla a cabo y en los tipos de hornos. $\mathrm{Y}$ los álcalis y el $\mathrm{SO}_{3}$ del clínker y del cemento influyen en el fraguado, en las resistencias, en la estabilidad de volumen y en la reacción expansiva árido-alcali. Todo ello de forma y en medida más o menos conocidas sobre las que no se va a tratar aquí.

Pero sí convendrá mencionar su relación con otro tema más típico aún, si cabe, de una Ciencia del Cemento: el de predecir de algún modo y con una cierta fiabilidad su comportamiento resistente.

Pues bien, entre los parámetros que condicionan la resistencia de un cemento a corto y largo plazo y sus cambios, están los álcalis y el $\mathrm{SO}_{3}$, y su distribución en el clínker.

Hasta el punto de que se pretende que éstos, y más concretamente los sulfatos alcalinos, permitan predecir el curso del endurecimiento de un cemento, y actuar debidamente cuando en una fábrica se presentan problemas acerca del mismo.

El primer hecho observado es el de que los sulfatos alcalinos del clínker influyen en la resistencia del cemento, en el sentido de que, hacen aumentar las de edades más cortas (pocos días) y disminuir a veces las de 28 días o posteriores, lo mismo que sucede con las 
adiciones de sulfato potásico al cemento en el agua de amasado, o con el tratamiento térmico del mortero. Esto autoriza a pensar que si los efectos son los mismos, los mecanismos por los que se producen - las reacciones de hidratación- también pueden serlo.

La determinación de los álcalis solubles del clínker incluye, no sólo los que se encuentran como sulfatos, sino también los que se hallan como $\mathrm{KC}_{23} \mathrm{~S}_{12}$ y $\mathrm{NC}_{8} \mathrm{~A}_{3}$.

La determinación directa e independiente de los que se encuentran en forma de sulfatos se puede hacer a partir de datos de análisis de $\mathrm{K}, \mathrm{Na}$ y $\mathrm{SO}_{3}$, por cálculo químico, o mediante curvas de calibrado. Estas permiten establecer que la cantidad de álcalis totales que se encuentra formando sulfato es el $80 \%$, y que la relación de sulfato potásico a sulfato sódico (expresado como sulfato potásico) es una función lineal de la relación de óxido potásico total a óxido sódico total (expresado como óxido potásico), cuyo coeficiente angular es $2(*)$.

Con la distribución de álcalis así establecida, con los módulos del clínker y con sus contenidos de $\mathrm{C}_{3} \mathrm{~S}$ y $\mathrm{C}_{2} \mathrm{~S}$ por una parte, y las resistencias a 3, 7 y 28 días (y la relación 28/3) por otra, se han llevado a cabo regresiones lineales múltiples que permiten, al parecer, predecir la resistencia a 28 días con una desviación típica de $25 \mathrm{~kg} / \mathrm{cm}^{2}$ (aproximadamente $5 \%$ ), variación que es normal en las fábricas. Esto puede permitir, tal vez, garantizar una cierta resistencia de un cemento, a partir de sus materias primas, o modificar dichas resistencias entre ciertos límites. Por ejemplo, un aumento del contenido de $\mathrm{K}_{2} \mathrm{SO}_{4}$ de $0,1 \%$ hace disminuir en $2 \%$ la resistencia a 28 días, pero aumenta las de 3 y 7 días, y esto se puede conseguir añadiendo yeso al crudo o añadiendo polvo de electrofiltro al cemento; lo contrario se conseguiría usando un combustible de contenido menor de azufre. Por otra parte, la resistencia media del cemento correspondiente a una determinada línea de producción se debe determinar en régimen de marcha estacionaria, con respecto a los contenidos de álcalis y $\mathrm{SO}_{3}$ del clínker, que deben ser los correspondientes a las materias primas, a la maquinaria y al proceso, y que permiten a su vez determinar el óptimo real de yeso del cemento, a efectos de fraguado, resistencia y retracción.

Es ésta, otra línea más de investigación que, encaminada por derroteros específicos, le es familiar al IETCC desde hace muchos años, y en la cual los demás —quiero decir la industria del cemento y la de maquinaria para la fabricación del mismo-, nos están dando la razón al demostrar con su interés y con su propia acción, que dista mucho de estar agotada, y que serán precisos aún años de trabajo para sacar de su estudio todo el mucho fruto que se puede y se debe obtener.

En el otro aspecto, en el técnico-económico, soluciones mejores que la creación de nuevas compañías, la cual es poco rentable y poco apetecible, pueden ser:

i) la fusión de firmas ( ¡ la cooperación y la racionalización!) para reducir costos y financiar nuevas instalaciones, así como la fusión de empresas cementeras y hormigoneras (de construcción);

ii) la construcción de grandes unidades y líneas de producción, más baratas por tonelada de capacidad instalada, bien localizadas, teniendo en cuenta factores ecológicos y ambientales (**);

iii) la transferencia de excedentes intermercados por transporte a granel (barco-tren), molienda "regional" o "comarcal" de clínker, e instalación de envasadoras y de silos de cemento estratégicos que se surtan de distintas procedencias.

\footnotetext{
(*) Todo esto quiere decir que un clínker con $1,875 \%$ de álcalis totales expresados como $\mathrm{K}_{2} \mathrm{O}$ tendría $1,500 \%$ de dichos álcalis en forma de sulfatos expresados como $\mathrm{K}_{2} \mathrm{SO}_{4}$ (de les cuales $1,000 \%$ sería $\mathrm{K}_{2} \mathrm{SO}_{4}$ y $0,500 \%$ sería $\mathrm{Na}_{2} \mathrm{SO}_{4}$ expresado como $\mathrm{K}_{2} \mathrm{SO}_{4}$ ), y el $0,375 \%$ restante estaría en forma de óxidos expresados como $\mathrm{K}_{2} \mathrm{O}$ (de los cuales $0,250 \%$ sería $\mathrm{K}_{2} \mathrm{O}$-en $\mathrm{KC}_{22} \mathrm{~S}_{12}$ - y $0,125 \% \mathrm{Na}_{2} \mathrm{O}$ expresado crma $\mathrm{K}_{2} \mathrm{O}$ —en $\mathrm{NC}_{8} \mathrm{~A}_{3}-$ ).

(**) Un inconveniente pueden ser los mayores costos de transporte que en el caso de fábricas menores y más dispersas.
} 
La competición en el dominio de mercados jugando a la baja de precios (téngase en cuenta el factor calidad) es desastrosa, tanto para el fabricante como para el consumidor.

Cuando una industria evoluciona a ritmo que sobrepasa en intensidad y rapidez lo previsible, después de un período de comercialización y promoción, surgen problemas para cuya solución hay que volver la vista al técnico de la producción y al hombre del laboratorio. Sostener el incremento de consumo en tales condiciones requiere de ambos: del uno, para mantener o aumentar, si es preciso, la capacidad de producción; del otro, para incrementar la promoción.

El aumento de la capacidad de producción puede carecer de incentivo por congelación de precios controlados, por exageradas e injustificadas exigencias medio-ambientales que elevan enormemente los gastos de instalación, por reducción de los suministros de combustible y/o energía a mayor precio, todo lo cual cae fuera del área de acción de la propia industria cementera.

Los efectos de un déficit de producción se podrían paliar restringiendo la promoción, pero ello perjudicaría permanentemente al cemento en el futuro, frente a otros materiales.

La solución de este planteamiento debe ser rápida y exige tener en cuenta que en el futuro no se va a tratar tanto de volumen de ventas, cuanto de producción rentable (¿competencia?). Ello implica considerar cuál va a ser la planta más económica; qué posibilidad habrá de reemplazar o modificar sistemáticamente el horno con precalentador, con algo más eficaz; cómo será el diseño de depuradores electrostáticos más eficaces y con menos averías; qué subproductos valdrá la pena recuperar y aprovechar, etc.

A cuenta de todo este planteamiento, hoy día no resuelto, los Estados Unidos importan en la actualidad cemento de diversos países, incluida España. El día que recojan este reto, el mercado internacional del cemento lo advertirá y las exportaciones españolas también. El ejemplo USA, con las dificultades de su industria cementera frente a su Administración en cuestiones de precios, energía, contaminación, seguridad, etc., puede ser aleccionador para nuestro país, y ya desde ahora en que aún es tiempo de evitar futuras dificultaides.

\section{CONCLUSIONES}

Los casos y aspectos considerados y tratados y los ejemplos expuestos, no son únicos ni exclusivos. Hay otros muchos cuya presentación exhaustiva sería tan prolija como ociosa.

Pero los que quedan de manifiesto bastan para demostrar que en el campo del cemento hay mucho y muy concreto por investigar.

Que no sólo este hecho en sí, sino su contenido, es decir, en qué, para qué y cómo hay que investigar, es algo que conocen bien, no sólo los investigadores "de oficio", sino los del ramo industrial de la fabricación y los del ramo industrial de la maquinaria para la misma.

Que existe, en general, una lógica —otros dirían insólita- comunión de criterios al respecto.

Que muchos de los temas caen, en opinion de todos, dentro de lo que, bien o mal, se suele llamar investigación básica - mirada por algunos con ciertos prejuicios o complejos-.

Pero que los ramos industriales antes mencionados no sólo no la desdeñan, sino que por sí mismos la sufragan y la llevan a cabo, dirlecta o indirectamente, convencidos de su rentabilidad primero, y de la imposibilidad de prescindir de ella, después, si se pretende incrementar, a cualquier plazo, el desarrollo y la promoción del cemento. 
Que muchos de los temas citados, no sólo no son de hoy, sino que son ya históricamente tradicionales; pese a lo cual "aún van a tener cuerda para rato", donde, cuando y mientras ello dependa de mentalidades y de decisiones objetivas, conscientes y responsables.

Y, finalmente, que en el estudio de buen número de estos temas el IETCC ha sido pionero, y hoy ve con satisfacción el refrendo que encuentra en la industria del cemento y afines -nacionales y extranjeras-, cuando se interesan por los mismos o análogos objetivos, hasta el punto de perseguirlos por sí mismas, y aplicando métodos y sistemas similares.

Un deseo y una esperanza finales: que tengan, que tengamos todos, ambiente y facilidades para lograr nuestras metas comunes.

R E F E R E N C I A S

Calleja, J.: “Consideraciones Generales Apologéticas sobre la Investigación Científica y Técnica".

Conferencia en el Auditorium del IMME (Instituto de Materiales y Métodos Estructurales) de la Universidad Central de Venezuela en Caracas, septiembre 1975, y en la sede de la Sociedad Científica Argentina en Buenos Aires, junio 1976.

Calleja, J.: "Lo que el futuro promete y exige al cemento y al Hormigón". IETCC, II Asamblea General, Madrid, noviembre 1974.

Calleja, J.: "Lo que el futuro promete y exije a los Aditivos para el Hormigón y a los productos para su tratamiento".

Cemento-Hormigón 46 (495), 599-607 (1975)

CEMBUREAU: Concrete Research Liaison Group, Wexham Springs (U.K.), octubre 1975.

CEMBUREAU: Technical Newsletter TN 4/1976: "Industrial International Data Base Pilot Study on the Cement Industry (Etude Pilote sur l'Industrie Cimentière)". Rapport du CCMS (Committee on the Challenges of Modern Society).

Davis, R. E. (Jr).: "Uniformity of Portland Cements: Facts and Fantasies". Modern Concrete, pág. 6163, abril 1975 .

IDoRN, G. M.: "Alkali-Silica Reaction" (Report).

CEMBUREAU Concrete Research Liaison Group. Report to the CEMBUREAU General Assembly, 1975.

Ishikawajima-Harima Heavy Industries, Co. Ltd.: "Oultine of IHI-SF Cement Clinker Burning Process". Tokyo (Japan), septiembre 1975.

JEPSEN, O. L.: (F. L. Smidth \& Co. Laboratories). "Effect of Alkalies and $\mathrm{SO}_{3}$ on the Strength of Cement and Clinker".

78th. Annual Meeting of American Ceramic Society, 1976.

JePsen, O. L. y Johansen, V. (F. L. Smidth \& Co. Laboratories). "Diffusion Controlled Reactions in Portland Cement Clinker".

78th. Annual Meeting of American Ceramic Society, 1976.

Johansen, V. y Jepsen, O. L. (F. L. Smidth \& Co. Laboratories). "Clinker Formation in Rotary Kilns". 78th. Annual Meeting of American Ceramic Society, 1976.

Koch-Petersen, Sv. Aa.: "Tendencias en la Industria del Cemento". Conferencia en la Asociación Brasileña del Cemento Portland. Sao Paulo (Brasil), 1971.

Le BeL, F.; Massazza, F.; IdoRn, G. M.: "Basic Research for the Development of Cement Markets". 
CEMBUREAU Concrete Research Liaison Group, Annex I to Circular Letter 71. Paris, 13 de octubre 1975.

MacLean, R. D.: Portland Cement, a Changing Industry".

Rock Products, págs. 92-95, enero 1973.

Mather, B.: "Le Rôle des Normes dans la Comunication Scientifique et Technique".

ASTM Standardisation News, julio 1975:

CEMBUREAU Technical Newsletter TN 2/1975.

Schroeder, E. W.: "The Future of the Cement Industry". Annual Convention of the National Ready Mixed Concrete Association.

Adelaide (Australia), octubre 1974.

Sfreda P. J. y Ramachandran, V. S.: "Predictability Gap between Science and Technology of Cements:

I Characterization of Hydraulic Cements".

Journal American Ceramic Society. 58, 3-4, 99-96 (1975).

Sereda, P. J. y Ramachandran, V. S.: "Predictability Gap between Science and Technology of Cements: II Physical and Mechanical Behaviour of Hydrated Cements".

Journal American Ceramic Society, 58, 5-6, 249-253 (1975). 


\section{COLOQUIO}

1. Abierto por el Presidente Sr. ARREDONDO lo inició el Sr. GASPAR para felicitar al ponente por su exposición y, para mencionar dentro del tema expuesto, la faceta de la formación de personal para el trabajo en los centros de investigación, haciendo al respecto una pregunta al Sr. CALLEJA acerca de lo que éste considera necesario para que dicho personal pueda rendir al máximo en centros de investigación aplicada.

Respondió el Sr. CALLEJA agradeciendo la pregunta y diciendo, en primer lugar, que en la exposición que había hecho no había tratado de todo el contenido de su extensa ponencia, y que en lo omitido por falta de tiempo había algo relacionado con la pregunta y que contestaba a ésta. En segundo lugar - añadió-, en dos ocasiones tuve la oportunidad de decir, al tratar en América del tema de la investigación, que el hombre es precisamente el sujeto activo de la misma, y que sin él, su vocación y su buena preparación no hay nada que hacer en ella, por mucho material, equipos y medios económicos de que se disponga. Efectivamente - prosiguió-, es necesario contar con investigadores lo mejor preparados, capaces, imaginativos y emprendedores posible, idea que materializo de forma caricaturesca, en el mejor de los sentidos —añadió-, diciendo que "el investigador debe ser capaz de limar con una sierra y de aserrar con una lima".

A mi juicio - prosiguió el Sr. CALLEJA-, la adecuada preparación de los investigadores de ciencia aplicada y de tecnología, la pueden y deben hacer, al más alto nivel de graduados, y después pasar éstos por todos los ciclos de la formación, los propios centros de investigación, en el campo específico de cada uno.

Y no sólo es necesaria esta preparación - continuó-, sino que también es absolutamente indispensable que el investigador la complemente, de manera aún más específica, en centros nacionales y extranjeros afines a los de su especialidad. Y, sobre esta base, sería también preciso que, después, se establecieran intercambios de investigadores, y de programas entre centros homólogos de países diferentes, para plantear y resolver de forma cooperativa problemas generales comunes, aportando cada cual sus ideas y sus peculiaridades. Esto se hace, y con éxito en general, pero no en la medida en que sería necesario hacerlo, porque del contraste y de la colaboración pueden salir mejor nuevas luces acerca de como orientar en el presente, y por supuesto en el futuro, la investigación en un campo determinado, según líneas de máximo interés general —científico, técnico y económico; o económico, técnico y científico, según las circunstancias-, establecidas en un orden prioritario.

En resumen -concluyó-, es imprescindible el investigador; éste tiene que tener la formación básica, complementaria y específica adecuadas; y todas ellas deben estar lo más actualizadas que sea posible en todo momento, por medio de reciclajes y demás recursos para tal efecto, los cuales deben ser arbitrados por los propios centros de investigación.

2. El Sr. PAZ intervino después felicitando al Sr. CALLEJA por su exposición, como Vicedirector del IETCC, sobre el futuro de la investigación en el campo del cemento y del concreto, ya al cierre de los Coloquios; así come también-al-Sr. ARREDONDO, por su disertación, como Director del Instituto, sobre el desarrollo de la industria del cemento, al comienzo de los mismos. Formuló después una pregunta acerca del estado actual de las investigaciones en Europa para la sustitución del yeso como regulador del fraguado del cemento. 
En respuesta, el Sr. CALLEJA dijo que la sustitución del yeso como regulador del fraguado del cemento era, como siempre, un problema específico de los países o zonas que carecen de él y se ven forzados a suplirlo con otros materiales adecuados a la función, los cuales, además, deben ser abundantes, baratos y fácilmente disponibles. Y que, en esa línea - continuó-, efectivamente se habrían hecho estudios para resolver el problema de la sustitución; y que, en virtud de los condicionantes mencionados, uno de los materiales en los que se había pensado era la caliza. Todavía no hay que yo sepa - dijo-, resultados definitivos al respecto, pero si puedo decir que las investigaciones siguen adelante, y que, naturalmente, no se van a limitar sólo al campo de los materiales más directos e inmediatos para una fábrica de cemento, sino que se van a extender a otros que, en principio, presentan ciertas posibilidades de éxito. Por ejemplo - prosiguió el Sr. CALLEJA-, en Japón se ha intentado utilizar - y creo recordar que acerca de ello se presentó algún trabajo en el Quinto Congreso Internacional de Química del Cemento de Tokyo en 1968, y en el sexto de Moscú de 1974-, algunos subproductos de naturaleza sulfática, procedentes de determinados procesos de fabricación de otras ramas industriales. Así, pues - concluyó-, materias naturales y subproductos artificiales están siendo ya investigados como sustitutivos del yeso en la regulación del fraguado del cemento.

3. El Sr. del CAMPO intervino después para decir que en la exposición hecha por el Sr. CALLEJA se habían puesto de manifiesto los amplios problemas científicos y técnicos que tiene planteados el campo del cemento a nivel de la investigación, y que constituían inquietudes bien captadas por el IETCC. Por la vinculación que entiendo que el Instituto tiene con la industria cementera española, me gustaría saber —dijo-, si ésta comparte esas inquietudes y si, en función de ello, hay posibilidad de aunar esfuerzos y actividades para abordar conjuntamente los problemas técnicos pendientes de solución, porque de no ser así —continuó-, va a ser difícil y desesperante para el investigador avanzar con provecho en el estudio de temas tan complejos y de tanta importancia para unos y para otros. La experiencia del Sr. CALLEJA al respecto, ¿cual es? -pregunto-.

El Sr. CALLEJA respondió que la opinión que en tal sentido interesaba más era la de la propia industria del cemento, globalmente considerada o tomada a nivel individual de empresas, puesto que la opinión del Instituto era bien clara y conocida de siempre, y que a ello respondían los hechos, no siempre bien divulgados y conocidos. Sin entrar ahora en pormenores, lo cual nos llevaría mucho tiempo -añadió-, creo que hay en la pregunta un aspecto importante: a mi juicio, el de la conveniencia -0 , mejor aún, necesidad-, de conocer en todo momento cuál es el criterio y la opinión de la industria cementera al respecto. Y para ello nada mejor que el diálogo y el intercambio de ideas y puntos de vista que no excluyen, sino que se complementan; para lo cual - dijo-, el Instituto está dispuesto siempre a través de todo su personal más capacitado a tales efectos, a ofrecer a la industria española del cemento su entusiasta colaboración en la solución de sus problemas, o al menos en el intento denodado de lograrlo, y más aún de cara al futuro.

El Sr. GASPAR, entrando más en el detalle del tema, como actual Jefe del Departamento de Química del IETCC, cargo en el que sucedió al Sr. CALLEJA, dijo que esa inquietud existe y no de ahora - aclaró-, sino desde hace años, por parte de determinadas industrias. $\mathrm{Y}$ que en ese sentido el Departamento venía realizando trabajos en colaboración con ellos, sobre ciertos problemas de su interés, cuya solución no es a corto plazo, sino a cinco y diez años vista - en el caso de algunos de dichos problemas-, y rebasando la escala de laboratorio para pasar a la escala semirreal. Añadió que se tropezaba, ahora tal vez más que nunca por la coyuntura económica, con la dificultad de la falta de personal y medios pero que, a pesar de ello, gracias a la colaboración, fruto del interés y de la inquietud de estas industrias, estaba siendo posible avanzar con provecho en las investigaciones emprendidas en común. 
4. A continuación el Sr. AGUANEL, en relación con el tema de la caliza utilizada como regulador del fraguado, admitió que estaba en estudio y sin resultados concretos por el momento. Pero quiero insistir - dijo-, en mi pregunta hecha ya en otra ocasión de estos Coloquios, acerca de si la caliza es activa o no. Si, según lo indicado por el $\mathrm{Sr}$. CALLEJA, puede tener una acción como regulador del fraguado, ello debe ser también porque pueda tener una actividad incluso química. De donde cabría deducir - concluyó el Sr. AGUANEL-, que la caliza no deberá ser considerada como una materia totalmente inerte, como un mero fíller, sino activa en una cierta medida, por un determinado tipo de reacción.

A esto respondió el Sr. CALLEJA que en el caso que se estaba tratando, de intentar utilizar la caliza en sustitución del yeso como regulador del fraguado del cemento, creía (y se expresaba así —dijo- por no estar seguro de ello, al no saberse aún gran cosa sobre el asunto), que era factible pensar en la formación de carboaluminatos, evidentemente por una interacción entre los aluminatos y la propia caliza. Que se forman carboaluminatos en el cemento hidratado, o durante la hidratación en determinadas condiciones -añadió-, es algo sabido y comprobado, si bien no siempre se conocen a fondo las cinéticas de los correspondientes procesos ni las condiciones óptimas o precisas para que dichas interacciones puedan tener lugar. Se habla - y se ha hablado en estos Coloquiosde "acciones epitáxicas" — dijo el Sr. CALLEJA—; el caso es —añadió-, que todo esto es lo que justamente constituye materia de estudio actual, con resultados finales no previsibles y que en su día llegarán a ser conocidos.

Y, aparte del caso de la caliza considerada como regulador del fraguado, si en la pregunta hecha por el Sr. AGUANEL va implícita la idea de una posible acción química, aparte de las físicas de las que ya se ha hablado en algún Coloquio anterior - dijo el Sr. CALLEJA-, pues tengo que decir que, en principio, no se puede excluir tal acción. basada posible y probablemente en la ya mencionada formación de carboalumiantos, puesto que está demostrado que éstos se originan por la acción del dióxido de carbono en la carbonatación atmosférica, y que en principio - volvía a repetir-, no había razón para excluir la posibilidad de un proceso con análogo final, a base de unas ciertas acciones topoquímicas -o epitáxicas, como en su momento dijo el Sr. DUTRON en un Coloquio pasado-, entre la caliza y los aluminatos del cemento.

Y no habiendo sido formuladas más preguntas, el Presidente, Sr. ARREDONDO, dió por concluido el Coloquio no sin antes dar las gracias al ponente y a los participantes en el mismo. 